\title{
Strong Consistency of CVaR Optimal Estimator
}

\author{
Xiaolin Li \\ School of Economic, Jinan University, Guangzhou, China \\ Email: jnulixiaolin@qq.com
}

How to cite this paper: Li, X.L. (2018) Strong Consistency of CVaR Optimal Estimator. Open Journal of Statistics, 8, 416-426.

https://doi.org/10.4236/ojs.2018.83027

Received: May 5, 2018

Accepted: May 25, 2018

Published: May 28, 2018

Copyright $\odot 2018$ by author and Scientific Research Publishing Inc. This work is licensed under the Creative Commons Attribution International License (CC BY 4.0).

http://creativecommons.org/licenses/by/4.0/

\begin{abstract}
Conditional Value-at-Risk ( $\mathrm{CVaR})$ is one of the commonly used risk measures. The paper shows that the optimal estimator of CVaR is strong consistency if the first-order moment of the population exists. We subsequently carry out numerical simulations to test the conclusion. We use the results to make an empirical analysis of Shenzhen A shares.
\end{abstract}

\section{Keywords}

Risk Measures, Conditional Value-at-Risk, Strong Consistency

\section{Introduction}

Because of inherent market instability, the ability to assess risk is very important so as to hedge against catastrophic loss. Therefore, some scholars have put forward the theory of value at risk $(\mathrm{VaR})$ and conditional value at risk (CVaR) theory as a form of risk mitigation.

To mitigate this risk, Markowitz [1] developed "expected returns-variance of returns" rule and illustrated geometrically relations between beliefs and choice of portfolio according to the rule. We find that is necessary to avoid investing in securities with high covariances among themselves, but we cannot calculate how much a set of investments might lose. In order to solve this problem, Guldimann, the head of JP Morgan global research department in 1993, pioneered the use of $\mathrm{VaR}$ in a report issued by the group of 30 . VaR theory has been gradually improved and widely applied in various fields of risk management, such as financial regulation, insurance industry and securities analysis. However, VaR has various undesirable conceptual and mathematical properties. Artzner et al. [2] [3] introduced a set of axioms for the proper construction of risk measures, including coherent measures of risk. The risk measure satisfies the transfer invariance, subadditivity, positive homogeneity and monotonicity. Therefore, cohe- 
rent measures of risk can be an effective tool for portfolio management. Then Rockafellar and Uryasev [4] put forward CVaR and found that CVaR can be more consisted measure of risk than VaR. In fact, $\mathrm{CVaR}$ can be thought of as the conditional expectation of losses that exceed the VaR (the definition of CVaR will appear in 2.1). Plung [5] proves that CVaR is a coherent risk measure and shows that $\mathrm{CVaR}$ is more effective than $\mathrm{VaR}$ in the risk management of the financial portfolios. Subsequently, CVaR theory is constantly improved and applied to real estate portfolio investment, supply chain management and so on. In order to seek to "cautious" predictions, Trindade et al. [6] proved the strong consistency of the CVaR optimization estimator and obtained the corresponding asymptotic distribution, under the condition that the second-order moment of population is limited. In this paper, we optimize the moment conditions of the above problems, and prove the strong consistency of CVaR optimal estimators under the condition that the first-order moment is finite.

The paper is organized as follows. In Section 2, we prove the strong consistency of $\mathrm{CVaR}$ optimal estimator under the finite first-order moment; we use $\mathrm{R}$ to simulate the correctness of the above conclusion in Section 3; in Section 4, we use the results to make an empirical analysis of Shenzhen A shares (A-shares trade on the two Chinese stock exchanges, the Shanghai Stock Exchange and the Shenzhen Stock Exchange. A-shares are shares of mainland China-based companies, and these shares were historically only available for purchase by mainland citizens because foreign investment was restricted).

\section{Theoretical Analyses}

\subsection{Risk Measurement: VaR and CVaR}

Let $Z$ be a random variable that represents a loss (or cost), $F(z)=P(Z \leq z)$ is the distribution function of $Z$. For $\alpha \in(0,1)$, the $\alpha$-quantile of $Z$ is defined as

$$
F^{-1}(\alpha):=\inf _{z \in R}\{z: F(z) \geq \alpha\} .
$$

In the context of monetary cost (loss), the $\alpha$-quantile $F^{-1}(\alpha)$ is a measure of risk commonly used in the finance industry, known as $\mathrm{VaR}$ and denoted $\operatorname{VaR}_{\alpha}(Z)=F^{-1}(\alpha)$. At this point, if $\mathrm{VaR}$ stands for the maximum loss, it can be exceeded only in $(1-\alpha) 100 \%$ of cases. And then CVaR, denoted by $\mathrm{CVaR}_{\alpha}(Z)$, can be thought of as the conditional expectation of losses that exceed the $\operatorname{VaR}_{\alpha}(Z)$ level. In case $\mathrm{Z}$ has a continuous distribution, $\mathrm{CVaR}_{\alpha}(Z)$ is given by the expectation of the right $(1-\alpha)$ tail of the cost distribution, that is

$$
\mathrm{CVaR}_{\alpha}(Z)=E\left[Z \mid Z \geq \operatorname{VaR}_{\alpha}(Z)\right] .
$$

Föllmer and Schied [7] simplify this model to be expressed as

$$
\operatorname{CVaR}_{\alpha}(Z)=\frac{1}{1-\alpha} \int_{\alpha}^{1} \operatorname{VaR}_{\gamma}(Z) \mathrm{d} \gamma,
$$

where is $[x]^{+}=\max \{0, x\}, x \in R$. 


\subsection{Strong Consistency of CVaR}

Let $Z$ is a random variable, $\left\{Z_{n}, n \geq 1\right\}$ is a Simple random from $Z$. Based on the definition of CVaR optimization proposed by Plung [5], Trindade et al. [6] introduced the following $\mathrm{CVaR}$ optimization estimator, i.e.,

$$
\hat{\theta}_{n}=\inf _{t \in R}\left\{t+\frac{1}{1-\alpha} \theta_{n}(t)\right\},
$$

where is $\theta_{n}(t)=n^{-1} \sum_{i=1}^{n}\left[Z_{i}-t\right]^{+}$. In addition, we define that

$$
\theta^{*}=\mathrm{CVaR}_{\alpha}(Z)=\inf _{t \in R}\left\{t+\frac{1}{1-\alpha} E[Z-t]^{+}\right\}, \theta(t)=E[Z-t]^{+} .
$$

It is obvious that $\theta_{n}(t)$ and $\theta(t)$ are monotonically decreasing and Lipschitz continuous. If $-\infty<s<t<+\infty$, that is

$$
\begin{gathered}
0 \leq \theta_{n}(s)-\theta_{n}(t)=\frac{1}{n} \sum_{i=1}^{n}\left(\left[Z_{i}-s\right]^{+}-\left[Z_{i}-t\right]^{+}\right) \leq t-s, \\
0 \leq \theta(s)-\theta(t)=E\left([Z-s]^{+}-[Z-t]^{+}\right) \leq t-s .
\end{gathered}
$$

Lemma 2.1. If $E|Z|<+\infty$ and $-\infty<a<b<+\infty$, then

$$
\lim _{n \rightarrow+\infty} \sup _{t \in[a, b]}\left|\theta_{n}(t)-\theta(t)\right|=0 \text { a.s. }
$$

Proof: For $\forall k \in R$, the intervals $[a, b]$ are divided into $k$ parts equally, where is $t_{j}=j(b-a) / k+a, j=0,1,2, \cdots, k, \theta_{n}(t)$ and $\theta(t)$ are monotonically decreasing and Lipschitz continuity, and $t_{j}-t_{j-1}=(b-a) / k$.

$$
\begin{aligned}
& \sup _{t \in[a, b]}\left|\theta_{n}(t)-\theta(t)\right|=\max _{1 \leq j \leq k} \sup _{t \in\left[t_{j-1}, t_{j}\right]}\left|\theta_{n}(t)-\theta(t)\right| \\
& =\max _{1 \leq j \leq k} \sup _{t \in\left[t_{j-1}, t_{j}\right]}\left|\theta_{n}(t)-\theta(t)-\theta_{n}\left(t_{j}\right)+\theta_{n}\left(t_{j}\right)-\theta\left(t_{j}\right)+\theta\left(t_{j}\right)\right| \\
& \leq \max _{1 \leq j \leq k}\left\{\sup _{t \in\left[t_{j-1}, t_{j}\right]}\left|\theta_{n}(t)-\theta_{n}\left(t_{j}\right)\right|+\sup _{t \in\left[t_{j-1}, t_{j}\right]}\left|\theta\left(t_{j}\right)-\theta(t)\right|+\left|\theta_{n}\left(t_{j}\right)-\theta\left(t_{j}\right)\right|\right\} \\
& \leq \max _{1 \leq j \leq k}\left\{\left(\theta_{n}\left(t_{j-1}\right)-\theta_{n}\left(t_{j}\right)\right)+\left(\theta\left(t_{j-1}\right)-\theta\left(t_{j}\right)\right)+\left|\theta_{n}\left(t_{j}\right)-\theta\left(t_{j}\right)\right|\right\} \\
& \leq \frac{2(b-a)}{k}+\max _{1 \leq j \leq k}\left|\theta_{n}\left(t_{j}\right)-\theta\left(t_{j}\right)\right|
\end{aligned}
$$

Applying the strong law of large number [8], it is easy to show that

$$
\lim _{n \rightarrow+\infty}\left|\theta_{n}\left(t_{j}\right)-\theta\left(t_{j}\right)\right|=0 \text { a.s. }
$$

for $1 \leq j \leq k$. We can get

$$
\lim _{n \rightarrow+\infty} \max _{1 \leq j \leq k}\left|\theta_{n}\left(t_{j}\right)-\theta\left(t_{j}\right)\right|=0 \text { a.s. }
$$

Therefore let $n \rightarrow+\infty$ and then let $k \rightarrow+\infty$, the conclusion can be get.

Lemma 2.2. If $E|Z|<+\infty$ and $b>0$, then

$$
\varlimsup_{n \rightarrow+\infty} \sup _{t>b}\left|\theta_{n}(t)-\theta(t)\right| \leq 2 E|Z| I_{\{Z>b\}} \text { a.s. }
$$

Proof: $\theta_{n}(t)$ and $\theta(t)$ is monotonically decreasing, we can get 


$$
\begin{aligned}
\sup _{t>b}\left|\theta_{n}(t)-\theta(t)\right| & \leq \sup _{t>b} \theta_{n}(t)+\sup _{t>b} \theta(t) \\
& =\sup _{t>b} \frac{1}{n} \sum_{i=1}^{n}\left[Z_{i}-t\right]^{+}+\sup _{t>b} E[Z-t]^{+} \\
& =\frac{1}{n} \sup _{t>b} \sum_{i=1}^{n}\left[Z_{i}-t\right]^{+}+\sup _{t>b} E[Z-t]^{+} \\
& =\frac{1}{n} \sum_{i=1}^{n}\left(Z_{i}-b\right) I_{\left\{Z_{i}>b\right\}}+E(Z-b) I_{\{Z>b\}} \\
& \leq \frac{1}{n} \sum_{i=1}^{n}\left|Z_{i}\right| I_{\left\{Z_{i}>b\right\}}+E|Z| I_{\{Z>b\}}
\end{aligned}
$$

Applying the strong law of large number, it is easy to show that

$$
\lim _{n \rightarrow \infty} \frac{1}{n} \sum_{i=1}^{n}\left|Z_{i}\right| I_{\left\{Z_{i}>b\right\}}=E|Z| I_{\{Z>b\}} \text { a.s. }
$$

So we can get the conclusion.

Lemma 2.3. If $E|Z|<+\infty$ and $a<0$, then

$$
\varlimsup_{n \rightarrow+\infty} \sup _{t<a}\left|\theta_{n}(t)-\theta(t)\right| \leq 2 E|Z| I_{\{Z<a\}} \text { a.s. }
$$

\section{Proof:}

$$
\begin{aligned}
& \sup _{t<a}\left|\theta_{n}(t)-\theta(t)\right| \\
& =\sup _{t<a}\left|\frac{1}{n} \sum_{i=1}^{n}\left[Z_{i}-t\right]^{+}-E[Z-t]^{+}\right| \\
& =\sup _{t<a}\left|\frac{1}{n} \sum_{i=1}^{n}\left(Z_{i}-t\right) I_{\left\{Z_{i} \geq t\right\}}-E(Z-t) I_{\{Z \geq t\}}\right| \\
& =\sup _{t<a}\left|\frac{1}{n} \sum_{i=1}^{n}\left(Z_{i}-t\right)\left(1-I_{\left\{Z_{i}<t\right\}}\right)-E(Z-t)\left(1-I_{\{Z<t\}}\right)\right| \\
& =\sup _{t<a}\left|\frac{1}{n} \sum_{i=1}^{n} Z_{i}-E Z-\frac{1}{n} \sum_{i=1}^{n}\left(Z_{i}-t\right) I_{\left\{Z_{i}<t\right\}}+E(Z-t) I_{\{Z<t\}}\right| \\
& \leq\left|\frac{1}{n} \sum_{i=1}^{n} Z_{i}-E Z\right|+\sup _{t<a} \frac{1}{n} \sum_{i=1}^{n}\left|Z_{i}-t\right| I_{\left\{Z_{i}<t\right\}}+\sup _{t<a} E|Z-t| I_{\{Z<t\}} \\
& \leq\left|\frac{1}{n} \sum_{i=1}^{n} Z_{i}-E Z\right|+\sup _{t<a} \frac{1}{n} \sum_{i=1}^{n}\left|Z_{i}\right| I_{\left\{Z_{i}<t\right\}}+\sup _{t<a} E|Z| I_{\{Z<t\}} \\
& =\left|\frac{1}{n} \sum_{i=1}^{n} Z_{i}-E Z\right|+\frac{1}{n} \sum_{i=1}^{n}\left|Z_{i}\right| I_{\left\{Z_{i}<a\right\}}+E|Z| I_{\{Z<t\}}
\end{aligned}
$$

Applying the strong law of large number, it is easy to show that

$$
\begin{gathered}
n^{-1} \sum_{i=1}^{n} Z_{i} \rightarrow E Z \text { a.s., } \\
n^{-1} \sum_{i=1}^{n}\left|Z_{i}\right| I_{\left\{Z_{i}<a\right\}} \rightarrow E|Z| I_{\{Z<a\}} \text { a.s. }
\end{gathered}
$$

So we can get the conclusion.

Proposition 2.1 If $E|Z|<+\infty$, then

$$
\lim _{n \rightarrow+\infty} \sup _{t \in R}\left|\theta_{n}(t)-\theta(t)\right|=0 \text { a.s. }
$$

Proof: Given $a$ and $b$ satisfied $-\infty<a<0<b<+\infty$, we can get 
$\sup _{t \in R}\left|\theta_{n}(t)-\theta(t)\right|=\max \left\{\sup _{t<a}\left|\theta_{n}(t)-\theta(t)\right|, \sup _{t \in[a, b]}\left|\theta_{n}(t)-\theta(t)\right|, \sup _{t>b}\left|\theta_{n}(t)-\theta(t)\right|\right\}$.

By applying Lemma 1, Lemma 2 and Lemma 3, we can get

$$
\varlimsup_{n \rightarrow \infty} \sup _{t \in R}\left|\theta_{n}(t)-\theta(t)\right| \leq \max \left\{2 E|Z| I_{\{Z<a\}}, 0,2 E|Z| I_{\{Z>b\}}\right\} .
$$

And let $a \rightarrow-\infty$ and $b \rightarrow+\infty$, we can get the conclusion.

Lemma 2.4. If $E[Z]^{+}<+\infty$, then

$$
\left|\hat{\theta}_{n}-\theta^{*}\right| \leq \frac{2}{1-\alpha} \sup _{t \in R}\left|\theta_{n}(t)-\theta(t)\right| .
$$

\section{Proof:}

$$
\begin{aligned}
& \left|\hat{\theta}_{n}-\theta^{*}\right|=\left(\hat{\theta}_{n}-\theta^{*}\right) I_{\left\{\hat{\theta}_{n}>\theta^{*}\right\}}+\left(\theta^{*}-\hat{\theta}_{n}\right) I_{\left\{\hat{\theta}_{n}<\theta^{*}\right\}} \\
& =\left(\inf _{t \in R}\left\{t+\frac{1}{n(1-\alpha)} \sum_{i=1}^{n}\left[Z_{i}-t\right]^{+}\right\}-\inf _{t \in R}\left\{t+\frac{1}{1-\alpha} E[Z-t]^{+}\right\}\right) I_{\left\{\hat{\theta}_{n}>\theta^{*}\right\}} \\
& +\left(\inf _{t \in R}\left\{t+\frac{1}{1-\alpha} E[Z-t]^{+}\right\}-\inf _{t \in R}\left\{t+\frac{1}{n(1-\alpha)} \sum_{i=1}^{n}\left[Z_{i}-t\right]^{+}\right\}\right) I_{\left\{\hat{\theta}_{n}<\theta^{*}\right\}} \\
& =\inf _{t \in R}\left\{t+\frac{1}{n(1-\alpha)} \sum_{i=1}^{n}\left[Z_{i}-t\right]^{+}-\inf _{t \in R}\left\{t+\frac{1}{1-\alpha} E[Z-t]^{+}\right\}\right\} I_{\left\{\hat{\theta}_{n}>\theta^{*}\right\}} \\
& \quad+\inf _{t \in R}\left\{t+\frac{1}{1-\alpha} E[Z-t]^{+}-\inf _{t \in R}\left\{t+\frac{1}{n(1-\alpha)} \sum_{i=1}^{n}\left[Z_{i}-t\right]^{+}\right\}\right\} I_{\left\{\hat{\theta}_{n}<\theta^{*}\right\}} \\
& :=I_{n 1}+I_{n 2}
\end{aligned}
$$

First, we can get

$$
\begin{aligned}
I_{n 1} \leq & \inf _{t \in R}\left|t+\frac{1}{n(1-\alpha)} \sum_{i=1}^{n}\left[Z_{i}-t\right]^{+}-\inf _{t \in R}\left\{t+\frac{1}{1-\alpha} E[Z-t]^{+}\right\}\right| \\
\leq & \inf _{t \in R} \mid t+\frac{1}{n(1-\alpha)} \sum_{i=1}^{n}\left[Z_{i}-t\right]^{+}-\left(t+\frac{1}{1-\alpha} E[Z-t]^{+}\right) \\
& +\left(t+\frac{1}{1-\alpha} E[Z-t]^{+}\right)-\inf _{t \in R}\left\{t+\frac{1}{1-\alpha} E[Z-t]^{+}\right\} \mid \\
= & \inf _{t \in R} \mid \frac{1}{n(1-\alpha)} \sum_{i=1}^{n}\left[Z_{i}-t\right]^{+}-\frac{1}{1-\alpha} E[Z-t]^{+} \\
& +\left(t+\frac{1}{1-\alpha} E[Z-t]^{+}\right)-\inf _{t \in R}\left\{t+\frac{1}{1-\alpha} E[Z-t]^{+}\right\} \mid \\
\leq & \sup _{t \in R}\left|\frac{1}{n(1-\alpha)} \sum_{i=1}^{n}\left[Z_{i}-t\right]^{+}-\frac{1}{1-\alpha} E[Z-t]^{+}\right| \\
& +\inf _{t \in R}\left|t+\frac{1}{1-\alpha} E[Z-t]^{+}-\inf _{t \in R}\left\{t+\frac{1}{1-\alpha} E[Z-t]^{+}\right\}\right| \\
= & \sup _{t \in R}\left|\frac{1}{n(1-\alpha)} \sum_{i=1}^{n}\left[Z_{i}-t\right]^{+}-\frac{1}{1-\alpha} E[Z-t]^{+}\right|=\frac{1}{1-\alpha} \sup _{t \in R}\left|\theta_{n}(t)-\theta(t)\right| .
\end{aligned}
$$

And then, we can get 


$$
\begin{aligned}
I_{n 2} \leq & \inf _{t \in R}\left|t+\frac{1}{1-\alpha} E[Z-t]^{+}-\inf _{t \in R}\left\{t+\frac{1}{n(1-\alpha)} \sum_{i=1}^{n}\left[Z_{i}-t\right]^{+}\right\}\right| \\
\leq & \inf _{t \in R} \mid t+\frac{1}{1-\alpha} E[Z-t]^{+}-\left(t+\frac{1}{n(1-\alpha)} \sum_{i=1}^{n}\left[Z_{i}-t\right]^{+}\right) \\
& +\left(t+\frac{1}{n(1-\alpha)} \sum_{i=1}^{n}\left[Z_{i}-t\right]^{+}\right)-\inf _{t \in R}\left\{t+\frac{1}{n(1-\alpha)} \sum_{i=1}^{n}\left[Z_{i}-t\right]^{+}\right\} \mid \\
= & \inf _{t \in R} \mid \frac{1}{1-\alpha} E[Z-t]^{+}-\frac{1}{n(1-\alpha)} \sum_{i=1}^{n}\left[Z_{i}-t\right]^{+} \\
& +\left(t+\frac{1}{n(1-\alpha)} \sum_{i=1}^{n}\left[Z_{i}-t\right]^{+}\right)-\inf _{t \in R}\left\{t+\frac{1}{n(1-\alpha)} \sum_{i=1}^{n}\left[Z_{i}-t\right]^{+}\right\} \mid \\
\leq & \sup _{t \in R}\left|\frac{1}{1-\alpha} E[Z-t]^{+}-\frac{1}{n(1-\alpha)} \sum_{i=1}^{n}\left[Z_{i}-t\right]^{+}\right| \\
& +\inf _{t \in R}\left|t+\frac{1}{n(1-\alpha)} \sum_{i=1}^{n}\left[Z_{i}-t\right]^{+}-\inf _{t \in R}\left\{t+\frac{1}{n(1-\alpha)} \sum_{i=1}^{n}\left[Z_{i}-t\right]^{+}\right\}\right| \\
= & \sup _{t \in R}\left|\frac{1}{n(1-\alpha)} \sum_{i=1}^{n}\left[Z_{i}-t\right]^{+}-\frac{1}{1-\alpha} E[Z-t]^{+}\right| \\
= & \frac{1}{1-\alpha} \sup _{t \in R}\left|\theta_{n}(t)-\theta(t)\right| .
\end{aligned}
$$

Hence, it is easy to show $I_{n 1}+I_{n 2} \leq 2 \sup _{t \in R}\left|\theta_{n}(t)-\theta(t)\right| /(1-\alpha)$. And we can obtain this conclusion.

Theorem 2.1. If $E|Z|<+\infty$, then $\hat{\theta}_{n} \rightarrow \theta^{*}$ a.s.

Proof: By applying Lemma 2.4 and Propostion 2.1, it is obvious that theorem is right.

\section{Numerical Simulation}

We carry out numerical simulations by using the standard t-distribution with 2 degrees of freedom (denoted $t(2)$ ), because the first-order moment of the distribution exists, and the second-order moment does not exist. Let $Z \sim t(2)$ and $\left\{z_{n}, n \geq 1\right\}$ be observations of $Z$. Andreev et al. [9] have mentioned that the true value of $C V a R$ is

$$
\mathrm{CVaR}_{\alpha}=2 \frac{f(q)}{F(-q)}\left(1+\frac{q^{2}}{2}\right)=\frac{1}{\sqrt{2}(1-\alpha)}\left(1+\frac{q^{2}}{2}\right)^{-1 / 2}
$$

where is that $q$ is the a-quantile of $F(x)$. In addition, Min et al. [10] get the value of CVaR optimal estimator, that is

$$
\hat{\theta}_{n}=\inf _{t \in R}\left\{t+\frac{1}{n(1-\alpha)} \sum_{i=1}^{n}\left[z_{i}-t\right]^{+}\right\}=\min _{1 \leq j \leq n}\left\{z_{j}+\frac{1}{n(1-\alpha)} \sum_{i=1}^{n}\left[z_{i}-z_{j}\right]^{+}\right\}
$$

Next, we use $R$ and the above two equations to calculate the true value of CVaR to compare it with the value of the optimized estimator.

Figure 1 is the line graph of CVaR estimations when the maximum sample 

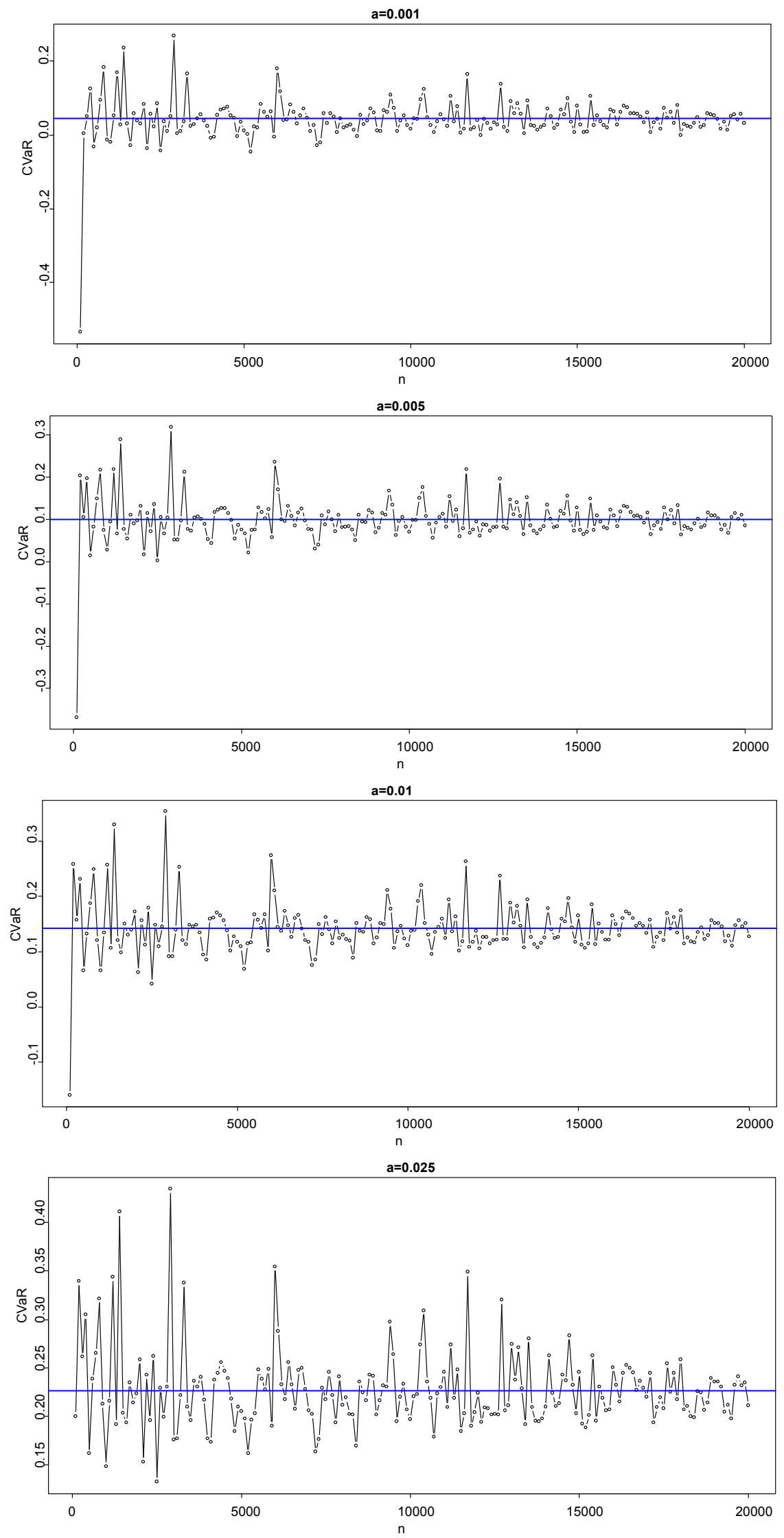


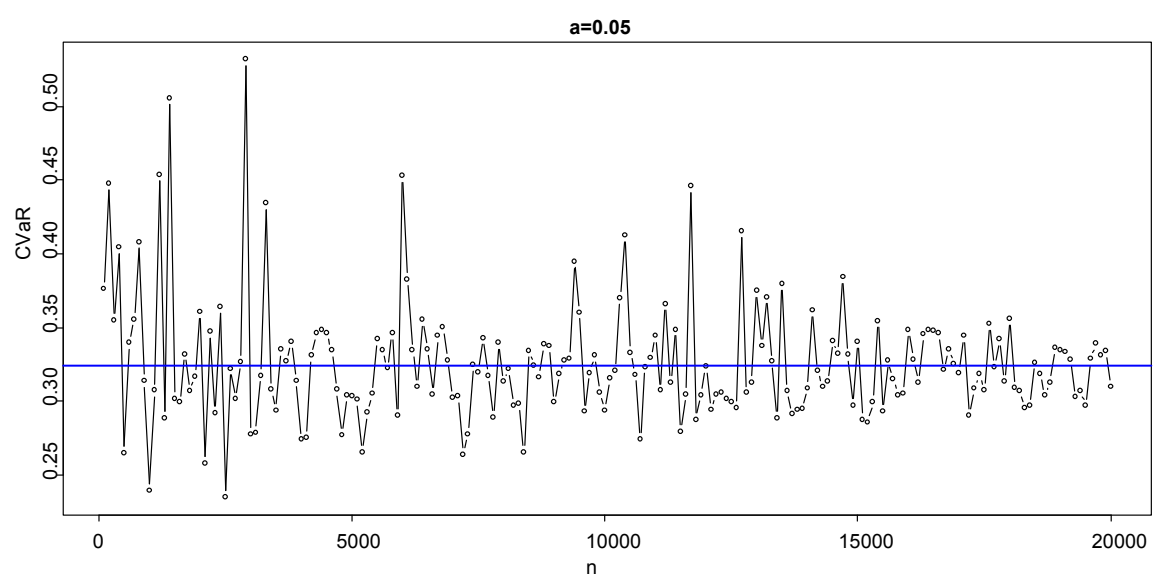

Figure 1. Simulation results when $\alpha$ takes different values.

size is $N=20000, \alpha$ is $0.001,0.005,0.01,0.025,0.05$ respectively and the simulated step length is 100. In Figure 1, the blue horizontal line represents the true value of $\mathrm{CVaR}$, and the points represent the estimated value of CVaR. So we can get the conclusion that when the sample size is the same, the smaller the value $\alpha$ is, the faster the convergence speed will be, the better the effect will be. For the $\alpha$, the CVaR estimations fluctuate on its true value; the fluctuation is smaller with the increase of sample size. It is easy to see that is less than 0.02 between the $\mathrm{CVaR}$ estimate and its real value when the number of sample $n$ satisfies $n \geq 10000$. In order to improve the accuracy of simulation, we continue to expand the sample size and get Table 1 eventually.

There are real valuations and estimations (obtained by numerical simulation) of CVaR under different $\alpha$ and $n$. From this table, we can see that difference between CVaR real valuations and estimations is $0.0312,0.025,0.0254,0.0255$, 0.0249 respectively when $n=10000$ and $\alpha=0.001,0.005,0.01,0.025,0.05$; but when $n=200000$, that difference is $-0.0024,-0.0034,-0.0038,-0.0045$, -0.0047 respectively.

Therefore we can get the conclusion that the larger the value is, the closer the estimation is to the real value. So we test the conclusion that the CVaR estimator has strong consistency.

\section{Empirical Analysis}

\subsection{Data Description}

In this Section, we use the results to make an empirical analysis of Shenzhen A shares, which included SWYA and Vanke A (two typical shares). We select a data range from November 27, 2006 to November 27, 2016 by R. Let the daily opening price of a single stock be $P_{O}$, and the daily closing one be $P_{C}$. We calculate the daily logarithmic yield of the stock $V$, i.e., $V=\ln P_{C}-\ln P_{O}$. In addition, let $L$ be the daily logarithmic loss rate of the stock. $\mathrm{CVaR}_{\alpha}(V)$ is CVaR of the stock's daily logarithmic yield rate at $\alpha$ level ; correspondingly, $\mathrm{CVaR}_{1-\alpha}(L)$ is the CVaR of the stock's logarithmic loss rate at $1-\alpha$ level. 
Table 1. CVaR estimations and real values.

\begin{tabular}{cccccc}
\hline \multirow{2}{*}{$n$} & \multicolumn{5}{c}{$\alpha$} \\
\cline { 2 - 5 } & 0.001 & 0.005 & 0.01 & 0.025 & 0.05 \\
\hline 10,000 & 0.0759 & 0.1258 & 0.1675 & 0.2520 & 0.3493 \\
20,000 & 0.0466 & 0.0993 & 0.1416 & 0.2274 & 0.3272 \\
30,000 & 0.0584 & 0.1088 & 0.1501 & 0.2355 & 0.3339 \\
40,000 & 0.0399 & 0.0962 & 0.1384 & 0.2226 & 0.3202 \\
50,000 & 0.0234 & 0.0805 & 0.1230 & 0.2078 & 0.3051 \\
60,000 & 0.0721 & 0.1265 & 0.1676 & 0.2503 & 0.3475 \\
70,000 & 0.0273 & 0.0830 & 0.1257 & 0.2110 & 0.3099 \\
80,000 & 0.0291 & 0.0878 & 0.1303 & 0.2141 & 0.3123 \\
90,000 & 0.0525 & 0.1084 & 0.1503 & 0.2341 & 0.3321 \\
100,000 & 0.0555 & 0.1106 & 0.1524 & 0.2375 & 0.3358 \\
120,000 & 0.0489 & 0.1026 & 0.1445 & 0.2290 & 0.3265 \\
140000 & 0.0439 & 0.0995 & 0.1413 & 0.2257 & 0.3241 \\
160000 & 0.0332 & 0.0911 & 0.1339 & 0.2181 & 0.3163 \\
180000 & 0.0504 & 0.1048 & 0.1463 & 0.2312 & 0.3293 \\
200000 & 0.0423 & 0.0969 & 0.1383 & 0.2220 & 0.3197 \\
Real valuation & 0.0447 & 0.1003 & 0.1421 & 0.2265 & 0.3244 \\
\hline & & & & & \\
\hline
\end{tabular}

The CVaR estimations of daily yield are calculated by

$$
\mathrm{CVaR}_{\alpha}(V)=\min _{1 \leq j \leq n}\left\{V_{j}+\sum_{i=1}^{n} \frac{\left[V_{i}-V_{j}\right]^{+}}{n(1-\alpha)}\right\} .
$$

Plung [5] suggest

$$
E V=(1-\alpha) \mathrm{CVaR}_{\alpha}(V)-\alpha \mathrm{CVaR}_{(1-\alpha)}(L) .
$$

\subsection{Empirical Analysis}

In this Section, we analyze the data of Vanke A and SWYA deeply. Figure 2 is the sequence diagram of 2589 daily logarithmic yield rate of Vanke A.

In this figure, the circle represents the real logarithmic yield of Vanke A, and the blue horizontal line represents the average logarithmic yield of the corresponding day. We can get the conclusion that real logarithmic yield of Vanke A always fluctuate around the average ones from this figure.

In addition, we can apply this property to invest. For example, we take Vanke $A$ and SWYA two stocks as an example to analyze. We get $\mathrm{CVaR}_{1-\alpha}(L)$ estimations of SWYA and Vanke A in Table 2 and Table 3. And we can get the average yield that SWYA 0.0027 and 0.0030 (Vanke A is $0.00209,0.00207$ ) respectively during nearly 10 years and nearly 5 years in Table 4 . Therefore, we can make a suitable portfolio based on our preference. 


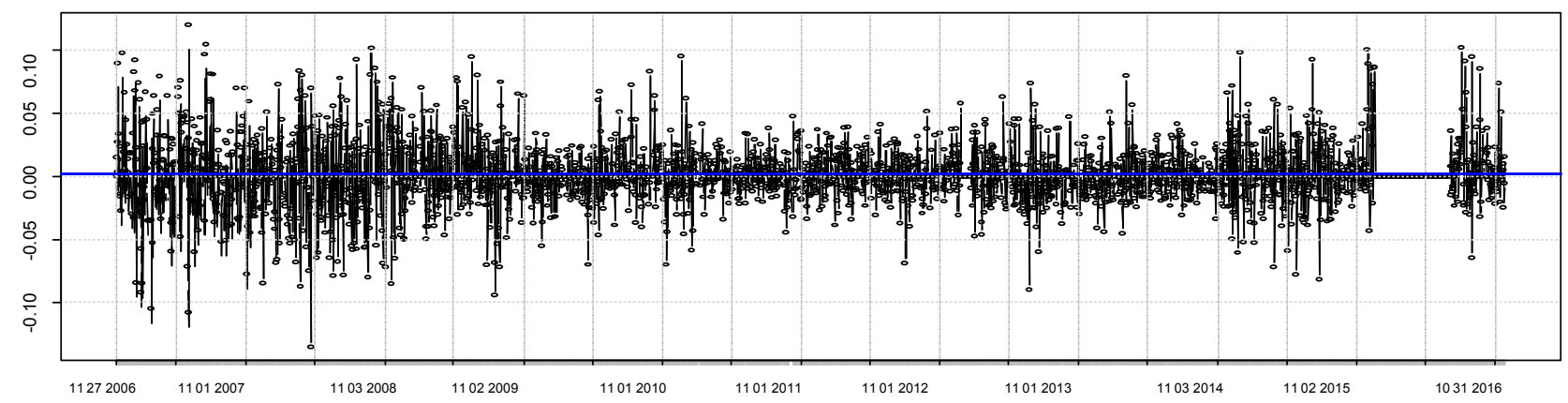

Figure 2. Daily logarithmic yield rate of Vanke A.

Table 2. $\mathrm{CVaR}_{1-\alpha}(L)$ estimation of Vanke A.

\begin{tabular}{cccccc}
\hline \multirow{2}{*}{ time/year } & \multicolumn{5}{c}{$\alpha$} \\
\cline { 2 - 6 } & 0.001 & 0.005 & 0.01 & 0.025 & 0.05 \\
\hline 10 & 0.1224 & 0.0986 & 0.0894 & 0.0762 & 0.0654 \\
5 & 0.0924 & 0.0795 & 0.0699 & 0.0562 & 0.0487 \\
\hline
\end{tabular}

Table 3. $\mathrm{CVaR}_{1-\alpha}(L)$ estimation of SWYA.

\begin{tabular}{cccccc}
\hline \multirow{2}{*}{ time/year } & \multicolumn{5}{c}{$\alpha$} \\
\cline { 2 - 6 } & 0.001 & 0.005 & 0.01 & 0.025 & 0.05 \\
\hline 10 & 0.1601 & 0.1152 & 0.1018 & 0.0860 & 0.0769 \\
5 & 0.1891 & 0.1306 & 0.1150 & 0.0951 & 0.0823 \\
\hline
\end{tabular}

Table 4. $\mathrm{CVaR}_{1-\alpha}(L)$ estimation of SWYA.

\begin{tabular}{ccc}
\hline Time/year & SWYA & Vanke A \\
\hline 10 & 0.0027 & 0.00209 \\
5 & 0.0030 & 0.00207 \\
\hline
\end{tabular}

\section{Conclusion}

In this article, we have proved that the optimal estimator of CVaR is strong consistency if the first-order moment of the population exists, subsequently carrying out numerical simulations to test the conclusion. Further, we will study the complete convergence and convergence rate of optimal estimator of CVaR.

\section{References}

[1] Markowitz, H.M. (1952) Protfolio Selection. Journal of Finance, 17, 77-91.

[2] Artzner, P., Delbaen, F., Eber, J.M., et al. (1997) Thinking Coherently. Risk, 10, 68-71.

[3] Artzner, P., Delbaen, F., Eber, J., et al. (1999) Coherent Measures of Risk. Mathematical Finance, 9, 203-228. https://doi.org/10.1111/1467-9965.00068

[4] Rockafellar, R.T. and Uryasev, S. (2000) Optimization of Conditional Value-at-Risk. Journal of Risk, 2, 21-41. https://doi.org/10.21314/JOR.2000.038 
[5] Plung, G.C. (2000) Some Remarks on the Value-at-Risk and the Conditional Value-at-Risk. In: Uryasev, S., Ed., Probabilistic Constrained Optimization, Springer, Berlin, 272-281.

[6] Trindade, A.A., Uryasev, S., Shapiro, A. and Zrazhevsky, G. (2007) Financial Prediction with Constrained Tail Risk. Journal of Banking and Finance, 31, 3524-3538. https://doi.org/10.1016/j.jbankfin.2007.04.014

[7] Föllmer, H. and Schied, A. (2014) Stochastics Finance-An Introduction in Discrete Time. 2nd Edition, De Gruyter, Berlin.

[8] Andreev, A. and Kanto, A. (2005) Conditional Value-at-Risk Estimation Using Non-Integer Values of Degrees of Freedom in Student's T-Distribution. Journal of risk, 7, 55-62. https://doi.org/10.21314/JOR.2005.108

[9] Jiang, M. and Meng, Z.Q. (2014) Multi Objective Conditional Value at Risk Theory. 1st Edition, Science Press, Beijing. (In Chinese)

[10] Wu, J.G. (2005) The Foundation of Modern Probability Theory. 2nd Edition, Fudan University Press, Shanghai. (In Chinese) 